as the most difficult in which to arrive at a diagnosis of the mental state. Of their cases they classify 2 males as alcoholic, 5 females as paranoic, 3 males and 1 female as delirious, 1 female and 2 males as maniacal, 3 females and 6 males as depressed.

Hereditary factors were present in $\mathbf{4 7}$ per cent of the series as a whole, and in 74 per cent of the cases in which a reliable history was obtained. Arteriosclerosis was recognised clinically in 10 cases and anatomically in 20 . There was marked general arteriosclerosis in 4 cases-3 were paranoics and 1 was maniacal. There was aortic sclerosis in 18 cases; 9 cases showed cerebral arteriosclerosis ; there was heart disease in 9, and kidney disease in 21 cases.

With regard to the correlation with atrophy of the brain, they note that atrophy of the brain is more marked in the females than among the males; but as the duration of life beyond the onset of the mental disease is also greater among the females, they naturally conclude that atrophy is a function of longevity rather than one of mental disease.

With regard to the distribution of the cortical pigments, the most interesting feature of their study was that the pigments were accumulated most largely in the bodies of the smaller nerve-cells in various cortical regions, perhaps more particularly in the suprastellate nerve-cell layers. Although it was probable that no brain was absolutely free from such pigmentation, yet 4 cases showed very little pigment in the nerve-cells. In 3 cases in which the precentral area showed excess of pigment, whereas the other areas were comparatively free, conduct disorder was a marked clinical fact. Perivascular cell pigmentation was a constant feature in all 23 cases. The lightest male and the lightest female brain showed only slight perivascular deposits. There was marked neuroglia cell pigmentation in alcoholic subjects.

From these facts the authors conclude that neither senile changes nor arterial changes have any necessary connection with the development of insanity in the sixth and seventh decades; that arteriosclerosis, senility, and the various forms of insanity are entities which frequently interpenetrate, but are logically and genetically quite separate. Perivascular cell pigments afford some index of the degree of faulty metabolism of the brain tissue; the neuroglial cell pigments vary rather with the age of the individual, and to some extent with the abuse of alcohol ; the nerve-cell pigments are subject to the greatest variation, and depend on some undetermimed principles.-R. M. MaRshaLL.

\title{
DISEASES OF THE SKIN.
}

A Study of Acnitis, with Report of an Extensive Case. By Dr. J. F. Schamberg (Journal of Cutaneous Diseases, January, 1909). - This communication is of special interest in view of the uncertainty as to the etiology of these rare cases, which have been described under a variety of names, amongst others, acne agminata (Crocker), acne telangiectodes (Kaposi), disseminated follicular lupus (Tilbury Fox), hydradenitis destruens suppurativa (Pollitzer). Dr. Schamberg's study of the literature, and his very complete histolngical, bacteriological, and experimental examination of his own case, lead him to the conclusion that the cause and vature of acnitis must meantime be set down as unknown. He sums up our present-day knowledge as follows :-

Barthélemy regards intestinal auto-intoxication as a factor. Most of the French dermatologists view acnitis and folliclis as identical, and look upon the affection as a tuberculide. Crocker says acnitis " is not in anyway connected with tuberculosis, either in the patient or family history."

A study of the subject appears to the author to strongly bear out the contention of Crocker.

The facts in favour of the tuberculous nature of the disease are the resemblance 
of the disease to folliclis, the occasional occurrence of tuberculosis in other members of the family, and the tuberculous aspect of the histological sections. Galloway and MacLeod, after a microscopic study of a growth in one of Perry's cases, remark that the evidence was undoubtedly in favour of the tubercular origin of the disease.

That the nodules of acnitis are not due to the organism of tuberculosis in the tissues is proven by the failure of all investigators to find tubercle bacilli in the nodules, and the negative result from inoculation of pus and fragments of nodules into guinea-pigs.

The absence of any definite reaction after the Calmette ocular tuberculin test, and the subcutaneous tuberculin injections in Dr. Schamberg's patient, makes it extremely unlikely that he is suffering from any tuberculous focus in the body.

The general tuberculous aspect of the sections of the nodules is bereft of much importance, since (Gilchrist has demonstrated that ordinary deep acne lesions may show upon microscopic study numerous giant cells, suggesting in appearance tubercular structure. The spontaneous and permanent cure of acnitis after its course is run is against the assumption of a tuberculous focus in the body liberating toxins, for the. disease appears to get well after the operating cause ceases to act.

From a consideration of the above facts, it would appear that there remains but little basis for the assumption that acnitis is of tuberculous origin. The character of the lesions, the course of the disease, and the microscopic picture suggest a parasitic granuloma. The failure to find the causative agent may be due to imperfect technique. There is also the possibility suggested by several writers that a toxic substance eliminated through the sweat glands may play an etiological rôle, but this hypothesis seems to Schamberg less likely.-J. W'yldie NicoL.

The Epithelium of the Buccal Mucosa. By Dr. W. Thorold Quaife (British Journal of Dermatology, December, 1908).-Dr Quaife undertook this investigation with the object of clearing up the question whether the buccal epithelium possesses a layer of cells containing keratin, it being frequently stated, especially in connection with leukoplakia of the tongue, that horny thickenings do occur. By buccal mucous membrane he means the epithelial of the mouth cavity, including that of the tongue, cheeks, and of the moist inner surface of the lips-the dry, red portion of the lips being epidermal. He studied the normal mucous membrane and also scrapings from patches of thickened mucosa from two cases-(1) a case of extensive leucoplakia buccalis of two years' duration; (2) a case of lichen planus. The following is a summary of his conclusions :-

1. The buccal mucosa resembles in its general structure the epidermis in possessing a proliferating basal layer of cells, the daughter-cells of which differentiate to form finally a layer of protective cells.

2. The daughter-cell appears in the later stages of its differentiation as a large cell with a small nucleus, scanty peripheral protoplasm, and, relatively, a large, clear perinuclear space.

3. No kerato-hyalin nor eleiden present.

4. In the deeper portion the intercellular lymphatic spaces are well marked and are crossed by numerous distinct "prickles."

5. The superficial protective layer is composed normally of nucleated cells smaller than epidermal horn cells, and these cells do not possess keratin "prickles" nor any form of keratin.

6. At the junction of the epidermis and the mucosa the change from the one to the other is usually very abrupt.

7. In certain pathological conditions of thickening of the mucosa the cells have advanced farther in their differentiation, resembling the epidermal horncell more closely in being larger and having no nucleus and possessing definite "prickles," which, however, are not of the nature of keratin. 